\title{
Editorial
}

\section{Technology and Psychology: The Application of VR Technology}

\section{Natasha Angelova*a}

[a] Department of Psychology, Faculty of Philosophy, South-West University "Neofit Rilski", Bulgaria.

\section{Abstract}

The development of technologies and their use in various fields of life and for different purposes have recently increased because of mandatory restrictive measures implemented by worldwide governments (to limit the spread of Covid-19). In this editorial are presented some notions about artificial reality and virtually created environments. It is discussed the opportunity to experience authentic emotions through virtual scenarios where people immerse themselves for teaching, training skills, or even when the real environment cannot be reproduced and only a simulated environment can help to enrich the experience and overcome some fears. It is shown that personal experience in a simulated reality, not only as an observer but also as a protagonist, stimulates and helps to acquire specific practical skills, and develop behavioral models leading to better learning and psychotherapeutic outcomes.

Keywords: Covid-19; VR; immersion; simulation; environment; artificial reality; psychology.

\section{Table of Contents}

VR technology and training

VR technology and the treatment of mental health problems

Limitations on using VR Technologies

Advantages of VR Technologies

References 
Psychological Thought, 2021, Vol. 14(2), 269-281, https://doi.org/10.37708/psyct.v14i2.656

Received: 2021-08-25. Accepted: 2021-09-30. Published (VoR): 2021-10-31.

Handling Editor: Irina Roncaglia, Chartered Practitioner Sport \& Exercise Psychologist, The National Autistic Society (NAS), United Kingdom.

*Corresponding author at: South-West University "Neofit Rilski", Blagoevgrad, Bulgaria. E-mail: natasha_v@swu.bg

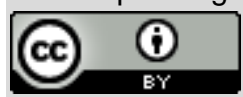

This is an open access article distributed under the terms of the Creative Common Attribution License (https://creativecommons.org/licenses/by/4.0), which permits unrestricted use, distribution, and reproduction in any medium, provided the original work is properly cited.

In 2020, the worldwide epidemiological situation resulting from Covid-19 provoked a reconsideration and reorganization of the daily life. This situation has affected both levels of people's individual and global needs in various areas of life such as economics, tourism, science, culture, and technology. From an industry and business point of view, staff's training was significantly limited, which also has led to a rethinking of the ways and methods of providing various forms of education, including universities and many research organizations. Adopting new approaches for organizing both personal and professional domains required more flexible way for interaction between people on one hand and between people and the environment on the other. The most used in these new conditions, was a remote form of interaction.

Hoping for a better way of dealing with the effects of the coronavirus crisis is the use of technologies. Until recently technologies were applied in a limited number of areas, mainly related to computer technology and its industry, or used in high-risk industries such as aviation, military training, creating models for interaction between different objects, by directly manipulating and constructing virtual reality for training in medicine and health. Since last year, technologies have been used and adopted more widely and included at everyday level.

In this sense, the use of virtual reality (VR) and immersion technologies for educational, therapeutic, research, tourism, design, and any other purposes has come to the fore as an opportunity to compensate for the lack of direct contact with one another that seems close to the physical reality, but it can still be done from a distance. The specific first-person perspective experience in immersion technologies, not only as an observer but also as a protagonist, stimulates the experience of acquiring specific knowledge or practical skills and 
support by developing behavioral models leading to the desired results (Fuchs et al., 2011). That makes the application of VR technology highly suitable for all purposes where physical distance is required. The realistic simulated environment allows people to immerse themselves in the atmosphere of a specific experience, which makes their reactions spontaneous (Gigante, 1993). The virtual environment gives people a unique opportunity to experience an authentic emotion related to the scenario they participate in, especially in situations where a real one cannot be reproduced. Through their direct experience, people perceive themselves directly as active participants in a simulated scenario, which challenges their resources and skills.

\section{Development of VR technologies}

The idea for the development of VR, however, may be related to the name of Morton Heilig, who, in his article from 1955 "The Cinema of the Future," revealed his idea for a machine that would be multi-dimensional and that would allow the viewer to immerse oneself in the virtual reality (Bouchard \& Rizzo, 2019). Heilig is a cinematographer who developed a machine called Sensorama (Srivastava et al., 2014). According to Heilig, the stories can be presented so that most of the viewers' senses are involved, stimulated, and accordingly, immersed in the new reality. In 1962, Heilig created a prototype of his machine and five short films to be shown through it.

The first device with a display placed on the head (head-mounted display), which most closely resembles modern VR glasses, was introduced by Philco Corporation in 1961 and was called Headsight (Srivastava et al., 2014).Through a small video screen and magnetic tracking, the display shows remote images that correspond to the direction of the head. It is primarily designed for use in dangerous situations, especially for training in military operations (Bouchard \& Rizzo, 2019; Nelson \& Yam, 2014).

Historically, the modern concept of VR is introduced in 1987 and mainly associated with the name of Jaron Lanier who owns a company for 3D graphics and computer games. He first started the production of modern equipment for creating and immersing in virtual reality (Berkman, 2018; Bouchard \& Rizzo, 2019). 
Modern VR technology allows a copy of reality through which the user's experiences can exceed the possibilities of the experiences in their own physical bodies. Oculus, Samsung, and Microsoft have the main credit for focusing public attention on VR because by improving modern technology, they manage to evoke a realistic experience much more tangible than before (Bown et al., 2017).

\section{VR technology and training}

Through VR immersive technologies, teachers are given the opportunity to create new learning content that is relevant to a particular training setting (Kaufman, 2003; Pantelidis, 2010; Youngblut, 1998).

VR technologies provide an opportunity to develop an innovative approach to encourage learners to participate more actively in the learning process. Through targeted scenarios, students are allowed to put themselves in the place of an active observer and, at the same time, a protagonist in the simulated virtual situation.

Learners can exercise the acquired knowledge in various areas such as the formation of presentation skills, conducting interviews and negotiations in more complex and dynamic situations, from public speaking, through medical procedures, to training to deal with extreme situations (Hsieh \& Lee 2018; Ku et al., 2007).

VR has successfully provided a safe environment for training students and trainees to manage stress and many other social skills (Bush, 2008; Hsieh \& Lee 2018; Miloff et al., 2016; Voss et al., 2016; Youngblut, 1998). VR is also used successfully to improve performance in professional sports, business, and art (Hsieh \& Lee 2018; Kaufman, 2003; Roussos et al., 1999; Wexelblat, 2014).

VR training can be done using one or more modes: Teacher mode, Normal tutorial, Autotutorial, Exam mode (Kaufman, 2003).

Through the first mode (Teacher mode), the teacher presents the overall topic or concept provided in the lesson and explains all the steps by showing in the virtual environment visual examples. The teacher can move forward in the lesson or go back to clarify the information, 
and answer questions. Several people can be trained simultaneously, in a shared room, or remotely located in different places.

In the second method (Normal tutorial), the whole presentation or various parts are "played" by actors or animated. The information and explanations are integrated into a specific scenario in which the learners actively participate and interact with the content. At the same time, the teacher navigates the students in their work and their interaction with the information. It is also possible to train several people at the same time, together or remotely.

The third type of VR training is through Auto-tutorial or Self-training. Learners go through various lessons on their own, in which they interact with the information. Initially, they can listen to a recording with instructions. The instructions can be voice recordings or texts for selfreading. The specificity of the recordings can also be focused on whether there are learners with specific educational needs, people with different kinds of disabilities, for example hearing impairments. Usually, learners receive instructions in self-training mode, see the situation visually, or interact with the information. That is the actual learning process, it requires several rehearsals and repetitions for a specific period as a way of transforming information into knowledge.

Exam mode offers learners the opportunity to present the content they have been trained on. Here the tasks are strictly specific according to the field - they can be textual, visual, construction. After completing the exam tasks, students must press a button to check their answers and compare them with the previously entered correct answers.

Most researches, in VR for training purposes, focus on problems in the use of software or applications, but not so much on its effectiveness for further training. According to Roussos et al. (1999), when using VR for educational purposes, additional researches in the following areas should be considered: technical, orientation-coordination, emotional, cognitive, and pedagogical.

- The technical aspects that are important to develop are: interface problems, physical problems, software, and hardware. 
- Problems related to orientation and coordination should focus on the user-virtual environment connection, including navigation, spatial orientation, sense of the present, level of immersion in the environment, and feedback.

- The emotional parameters that are good to explore are the user's involvement in the experience, liking or rejecting the virtual environment, confidence in the virtual environment.

- The cognitive aspect of the research should be aimed at studying the change in cognitive abilities and skills through immersion in VR interaction and learning.

- The pedagogical aspect refers to the teaching approach. The aim is to find the best way to present the subject matter in relation to the field, the context of the situation, and the objective physical capabilities of the learners.

\section{VR technology and the treatment of mental health problems}

VR technologies are also often used to treat psychosomatic, anxiety, phobic, and depressive states (Andrews et al., 2018; Bouchard et al., 2006; Eichenberg, 2011). By practicing in a simulated reality, the change in social behavior is facilitated by the real-time study of the factors influencing behavior. The application of VR for psychotherapeutic purposes has two main parts: the first is developing diagnostic scenarios, through which the issue and the patient's reactions to the issue could be studied. For this aim, the scenarios are developed with an appropriate protocol that follows the logic of the specific therapy or intervention in the examination of the patient and allows smooth movement to the next second part of the application of VR technologies for therapy, namely building skills to accept and implement change.

The realization of a change in a psychotherapeutic setting, according to Greenberg \& Safran (1987 in Bouchard et al., 2012), has two main directions: bottom-up and top-down. For people who process information from the bottom up, the specific unlocking moment begins with an emotional experience and leads to changes in behavior and concepts. In people with top-down processing of the issue the change is related to the study of concepts and beliefs, which leads them to consider their emotional reactions and plan an alternative and more adaptive behavioral response. In this context, respectively to the therapeutical and ethical protocol, in 
virtuo scenario can be used to trigger specific fear response by exposing stimuli to the patient (Bouchard et al., 2012). In this way, the VR situation helps the therapist reach specific memories or discover specific patterns of selective attention or automatic thoughts that provoke a particular reaction. On the other hand, virtual scenes can help the patient isolate themselves from the outside world and train specific actions or play different scenarios, which will prepare them for a future encounter with the stress stimulus. Neubeck and Neubeck (1998) suggested that VR stimuli can also be symbolic material to lead the patient to awaken and release deeper experiences, including traumatic ones that can be associated with diffuselybuilt fictitious worlds and analyzed in a usual therapeutical setting.

More generally, VR immersion enables patients to experience, in a safe environment where they can control the access to the stimulus, life events that are emotional for them or have a specific value in their lives, but for which they cannot cope or respond adequately. In order the effects of the therapy to be lasting, patients must continue their attempts to expose themselves to the fearful stimulus in a real environment and even overcome it by maintaining regular contact behavior with it for a certain period. Of course, the latter applies to patients with phobic conditions, in which therapy requires exposure to the stimulus and regular contact with it, such as fear of flying, agoraphobia, etc.

For the specialist, VR is a helpful tool for presenting to the patient the ability to distinguish the stimulus that causes fear from the thoughts and beliefs attached to it. This changes the perception of the stimulus and allows to change the patient's behavioral response and to learn new reactions and adaptive responses to the stimulus. VR usage for psychotherapeutic purposes is predicted as one of the most popular methods in counseling and therapy for 2022 (Norcross et al., 2013).

\section{Limitations on using VR Technologies}

A disadvantage in using VR technologies in psychology is that there are no standardized protocols for studying the mental health issues and protocols for working on every specific condition. Another serious difficulty is the lack of standardization of VR software and the limited possibilities to build the therapeutic setting in the virtual environment. Finally, there is 
the high price of VR equipment and the design of the scenarios for each specific issue. However, the main limitation of this method is that it still offers a virtual reality of which the patient is informed and aware. Experiences during stimulation and the successes achieved in the therapeutic process remain in a separate reality until they are integrated into the patient's real life.

Other disadvantages of VR immersion that should also be considered are related to the negative/side effects of using the technique: visual (blurred vision, headache); disorientation (dizziness, vertigo); seasickness (nausea, vomiting) (Bouchard et. al., 2012). Therefore, the behavior of the trainer or therapist in the specific situation is critical. It is essential to warn VR users that some of the symptoms mentioned above could be experienced during immersion in virtual reality. It would also be a good idea for the trainer or therapist to warn that turning the head and gaze in different directions too often and too quickly in the virtual space could both arouse the side effects and deepen them (Carvalho et al., 2017; Wilson \& Soranzo, 2015).

\section{Advantages of VR Technologies}

The advantage of VR in allowing participants to engage in a particular scene is related to the ability of participants to maintain a sense of reality and respond to stimuli as if they were really "there." A sense of reality is crucial for participants to immerse themselves in the virtual environment to work out their assigned tasks. On the other hand, the immersion in VR itself is related to the technical parameters of the VR system and the details included in the scene.

Another advantage of VR in training for business, industrial, scientific, and university purposes is that it allows work from a distance. This advantage, viewed through the prism of the last year and more specifically the Covid-19 pandemic, is significant because it gives hope for further development in these sectors. VR immersion used for training creates opportunities to modernize traditional learning practices, which positively impacts many other aspects of life as it sets the mind free from concepts related to physical limitations. 
It is important to note the positive emotional outcomes for those who immerse in the virtual scenario. People like to play games and have fun, so the virtual experience gives them this opportunity. Increasing the motivation to learn and train new skills to overcome potential fears or phobic responses through play is part of the VR immersive game benefits. The immersion in VR could be useful, positive, and ultimately enjoyable. The pleasurable attitude of the participants while interacting in VR could be wisely used to improve their lives.

\section{Conclusion}

In summary, VR immersion technology is attracting increasing attention due to the physical constraints imposed by governments around the world in connection with the Covid-19 pandemic and guidelines related to limiting the spread of coronavirus. The applications of these technologies are more widely used than discussed here and overcome many of the difficulties caused by the Covid-19 pandemic in education, psychotherapy, and trainings in health care and medicine.

\section{Funding/Financial Support}

The author has no funding to report.

\section{Other Support/Acknowledgement}

The author has no support to report.

\section{Competing Interests}

The author is a member of the Editorial board of Psychological Thought. 


\section{References}

Andrews, G., Basu, A., Cuijpers, P., Craske, M.G., McEvoy, P., English, C.L. \& Newby, J.M. (2018). Computer therapy for the anxiety and depression disorders is effective, acceptable and practical health care: An updated meta-analysis. Journal of Anxiety Disorders, 55, 70-78. https://doi.org/10.1016/j.janxdis.2018.01.001.

Berkman, M. I. (2018). History of Virtual Reality. In N. Lee (Ed.), Encyclopedia of Computer Graphics and Games. Springer. https://doi.org/10.1007/978-3-319-08234-9_169-1

Bouchard, S., Côté, S., \& Richard D.S. (2006). Virtual reality applications of exposure. In D.S. Richard, \& D.Lauterbach (Eds.), Handbook of exposure therapies (pp. 347-388). Academic Press.

Bouchard S., \& Rizzo A.. (2019) Applications of virtual reality in clinical psychology and clinical cognitive neuroscience - An introduction. In A.Rizzo, \& S. Bouchard (Eds.), Virtual Reality for Psychological and Neurocognitive Interventions. Virtual Reality Technologies for Health and Clinical Applications (pp. 1-13). Springer. https://doi.org/10.1007/978-1-4939-9482-3_1

Bouchard, S., Robillard, G., Larouche, S., \& Loranger, C. (2012). Description of a treatment manual for in virtuo exposure with specific phobia. In C. Eichenberg (Ed.), Virtual Reality in Psychological, Medical and Pedagogical Applications (pp. 82-108). InTech.

Bown, J., White, E., \& Boopalan, A. (2017). Looking for the ultimate display: A brief history of virtual reality In J. Gackenbach, \& J. Bown (Eds.), Boundaries of Self and Reality Online (pp. 239-259). Academic Press. https://doi.org/10.1016/B978-0-12-804157-4.00012-8.

Bush, J. (2008). Viability of virtual reality exposure therapy as a treatment alternative. Computers in Human Behavior, 24(3), 1032-1040. https://doi.org/10.1016/j.chb.2007.03.006

Carvalho P., Miyagawa T., Maciel F., \& Melo P. (2017) VR Rio 360: The challenges of motion sickness in vr environments. In S. Lackey \& J. Chen (Eds.), Virtual, Augmented and Mixed Reality. Lecture Notes in Computer Science, vol. 10280 (pp. 495-504). Springer. https://doi.org/10.1007/978-3-319-57987-0_40

Eichenberg, C. (2010). Application of virtual realities in psychotherapy: possibilities, limitations and effectiveness. In Jae-Jin Kim (Ed.), Virtual Reality (pp. 469-484). InTech. Available from: 
http://www.intechopen.com/books/virtual-reality/application-of-virtual-realities-in-psychotherapypossibilities-limitations-and-effectiveness

Fuchs Ph., Moreau, G., \& Guitton P. (2011). Virtual Reality: Concepts and Technologies. CRC Press. https://doi.org/10.1201/b11612

Gigante, M. A. (1993). Virtual reality: definitions, history and applications. In R.A. Earnshaw, M.A. Gigante, \& H. Jones (Eds.), Virtual Reality Systems (pp. 3-14). Academic Press. https://doi.org/10.1016/B978-0-12-227748-1.50009-3

Greenberg, L. S., \& Safran, J. D. (1987). Emotion in psychotherapy: Affect, cognition, and the process of change. Canadian Psychology/Psychologie canadienne, 49(1).

Hsieh, M. C., \& Lee, J. J. (2018). Preliminary study of VR and AR applications in medical and healthcare education. Journal of Nursing and Health Studies,3(1:1). http://www.imedpub.com/nursing-andhealth-studies/

Kaufmann, H. (2003). Collaborative augmented reality in education. Institute of Software Technology and Interactive Systems, Vienna University of Technology. [Link]

Ku, J., Han, K., Lee, H.R., Jang, H.J., Kim, K.U., Park, S.H., et al. (2007). VR-based conversation training program for patients with schizophrenia: A preliminary clinical trial. Cyberpsychology \& Behavior, 10(4). 567-574. https://doi.org/10.1089/cpb.2007.9989

Miloff, A., Lindner, P., Hamilton, W., Reuterskiöld, L., Andersson, G., et al. (2016) Single-session gamified virtual reality exposure therapy for spider phobia vs. traditional exposure therapy: Study protocol for a randomized controlled non-inferiority trial. Trials, 17(1), 1-8. https://trialsjournal.biomedcentral.com/articles/10.1186/s13063-016-1171-1

Nelson. F. \& Yam, M. (2014). The past, present, and future of VR and AR: The pioneers speak. Tom's Hardware, 3811-3813.com [Link]

Neubeck, A. K., \& Neubeck, B. (1998) Virtual reality as a support system for psychodynamic treatment. Cyberpsychology \& Behavior 1(4). 341-345. https://doi.org/10.1089/cpb.1998.1.341

Norcross, J. C., Pfund, R. A., \& Prochaska, J. O. (2013). Psychotherapy in 2022: A Delphi poll on its future. Professional Psychology: Research and Practice, 44(5), 363-370. https://doi.org/10.1037/a0034633 
Pantelidis, V. S. (2010). Reasons to use virtual reality in education and training courses and a model to determine when to use virtual reality. Themes in Science and Technology Education, 2(1-2), 5970. https://eric.ed.gov/?id=EJ1131313

Roussos, M., Johnson, A., Moher, T., Leigh, J., Vasilakis, C., \& Barnes, C. (1999). Learning and building together in an immersive virtual world. Presence: Teleoperators and Virtual Environment 8(3). 247-263. https://doi.org/10.1162/105474699566215

Srivastava, K., Das, R. C., \& Chaudhury, S. (2014). Virtual reality applications in mental health: Challenges and perspectives [Editorial]. Industrial Psychiatry Journal, 23, [serial online].83-85. [cited 2021 Aug 13] https://www.industrialpsychiatry.org/text.asp?2014/23/2/83/151666

Voss, C., Washington, P., Haber, N., Kline, A., Daniels, J., et al. (2016) Superpower glass: delivering unobtrusive real-time social cues in wearable systems. In Proceedings of the 2016 ACM International Joint Conference on Pervasive and Ubiquitous Computing: Adjunct. 1218-1226. https://arxiv.org/ftp/arxiv/papers/2002/2002.06581.pdf

Wexelblat., A. (2014). Virtual reality: Applications and explorations. Academic Press.

Wilson, C. J., \& Soranzo, A. (2015). The use of virtual reality in psychology: A case study in visual perception. Computational and Mathematical Methods in Medicine. e151702. https://doi.org/10.1155/2015/151702

Youngblut, C. (1998). Educational Uses of Virtual Reality Technology. Institute for Defense Analyses Alexandria VA. https://apps.dtic.mil/sti/pdfs/ADA339438.pdf

\section{About the Author}

Natasha Virmozelova Angelova Ph.D. is an Associate Professor at the Department of Psychology at SWU "Neofit Rilski", Blagoevgrad, Bulgaria. Her interests are in the areas of Health Psychology, Psychological Researches, Stress and Coping Strategies, Consultative Psychology and Psychotherapy, Cognitive Psychology, Psychology of Family Relationships. From 2003 to 2007 she worked at the Psychology Laboratory at the Institute of Psychology of the Ministry of the Interior. She is an Editor-in-chief of Psychological Thought and an editor in Yearbook of Psychology, released by SWU "Neofit Rilski", Blagoevgrad, Bulgaria. 


\section{Corresponding Author's Contact Address ${ }^{[T O P]}$}

Department of Psychology,

South-West University "Neofit Rilski”,

Blagoevgrad, Bulgaria.

Email: natasha_v@swu.bg 